\title{
[Supplementary Materials]
}

\section{Theoretical Study on Drum-shaped Polymers (1,3,5-triazine) ${ }_{2 n}$ Composed of}

Nonplanar $\boldsymbol{\pi}$-extended Polymerization Units

Bing He, ${ }^{\dagger}$ Hongwei Zhou, $*, \dagger$

College of Chemistry and Life Science, Institute of Functional Molecules, Chengdu Normal University, Chengdu 611130, P.R. China

Correspondence to: Hongwei Zhou (E-mail: jcbzhou@cdnu.edu.cn) 

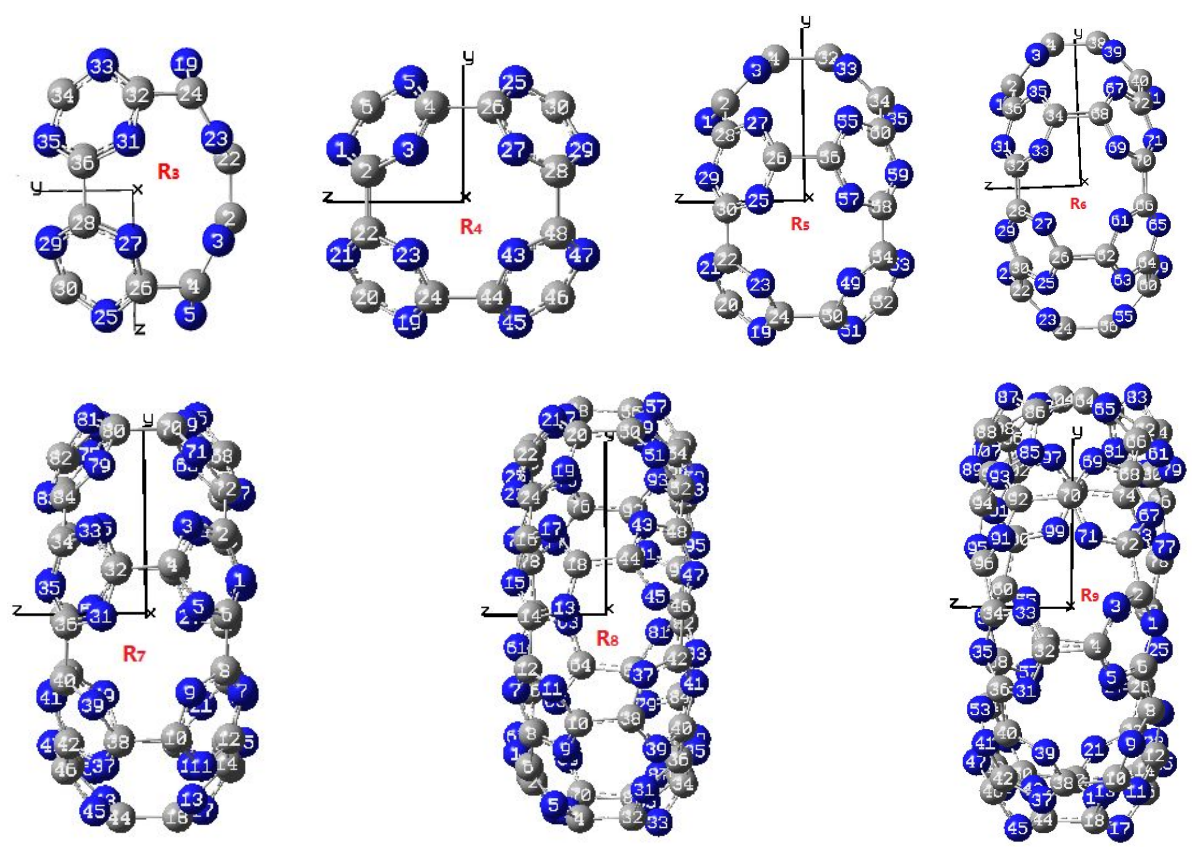

Figure S1. The $x$-axis views of optimized structures of $\mathbf{R}_{\boldsymbol{n}}$ at the B3LYP/cc-pvDZ level. $\mathbf{R}=\left(\mathrm{C}_{3} \mathrm{~N}_{3}\right)_{2}, \boldsymbol{n}=3-9$.

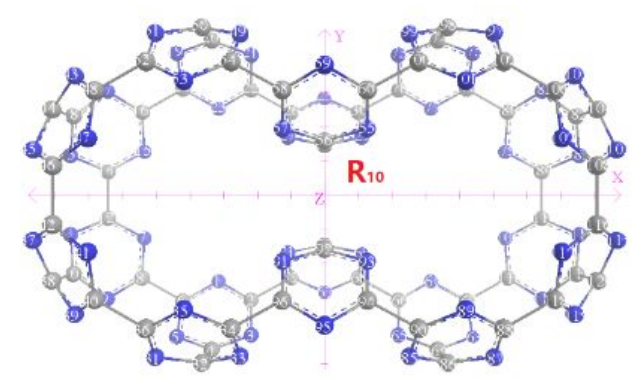

Figure S2. The $z$-axis view of optimized structure of $\mathbf{R}_{10}$ at the B3LYP/cc-pvDZ level. $\mathbf{R}=\left(\mathrm{C}_{3} \mathrm{~N}_{3}\right)_{2}$.

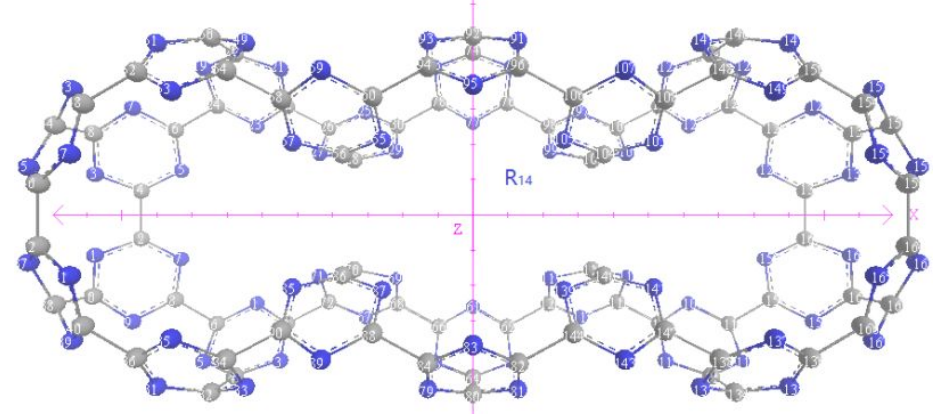

Figure S3. The $z$-axis view of optimized structure of $\mathbf{R}_{14}$ at the B3LYP/cc-pvDZ level. $\mathbf{R}=\left(\mathrm{C}_{3} \mathrm{~N}_{3}\right)_{2}$. 


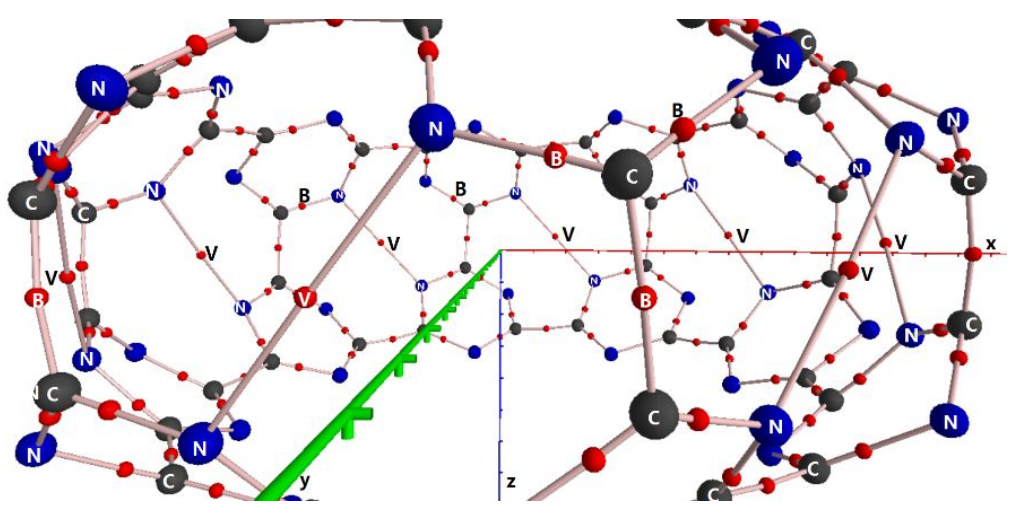

Figure S4. The $3 \mathrm{~d}$ view of the example polymer $\mathbf{R}_{\mathbf{8}}$ in AIM window at the B3LYP/cc-pvDZ theory level. $\mathbf{R}=\left(\mathrm{C}_{3} \mathrm{~N}_{3}\right)_{2}$. Three diagram elements of atoms, bond critical points (BCPs, small balls), and bond paths (lines) are shown. All the eight BCPs with $\nabla^{2} \rho>0$ are labelled by "V", parts BCPs with $\nabla^{2} \rho<0$ are labelled by "B". Only part of the geometric structure of $\mathbf{R}_{\mathbf{8}}$ can be displayed due to the limitation of AIM window space.

Table S1. The symmetries of $\mathbf{R}_{\boldsymbol{n}}$ at the stages of initial, post-optimality (PO) and approximate Higher-order-point group (AH) at the specified threshold values. $\mathbf{R}=\left(\mathrm{C}_{3} \mathrm{~N}_{3}\right)_{2}, \boldsymbol{n}=3-9$.

\begin{tabular}{cccc}
\hline polymer & Initial & $\mathrm{PO}($ threshold $\AA)$ & $\mathrm{AH}($ threshold $\AA)$ \\
\hline $\mathbf{R}_{3}$ & $C_{l}$ & $D_{3 h}(0.001)$ & $D_{3 h}(0.01)$ \\
$\mathbf{R}_{4}$ & $C_{I}$ & $O_{h}(0.001)$ & $O_{h}(0.01)$ \\
$\mathbf{R}_{5}$ & $C_{l}$ & $D_{5 h}(0.001)$ & $D_{5 h}(0.01)$ \\
$\mathbf{R}_{6}$ & $C_{l}$ & $D_{6 h}(0.001)$ & $D_{6 h}(0.01)$ \\
$\mathbf{R}_{7}$ & - & $C_{l}(0.01)$ & $C_{2}(0.03)$ \\
$\mathbf{R}_{8}$ & - & $C_{l}(0.001)$ & $D_{8}(0.01)$ \\
$\mathbf{R}_{9}$ & - & $C_{l}(0.01)$ & $C_{l}(0.05)$ \\
\hline
\end{tabular}

Table S2 the lowest three frequencies (in $\mathrm{cm}^{-1}$ ) of $\mathbf{R}_{\boldsymbol{n}}$ at the B3LYP/cc-pvDZ theory level. $\mathbf{R}=\left(\mathrm{C}_{3} \mathrm{~N}_{3}\right)_{2}, \boldsymbol{n}=3-10$.

\begin{tabular}{cccc}
\hline polymer & Frequency $\mathbf{1}$ & Frequency $\mathbf{2}$ & Frequency $\mathbf{3}$ \\
\hline $\mathbf{R}_{3}$ & 91.0178 & 160.9971 & 160.9971 \\
$\mathbf{R}_{4}$ & 105.0542 & 105.0542 & 140.1327 \\
$\mathbf{R}_{5}$ & 79.1780 & 97.6001 & 97.600 \\
$\mathbf{R}_{6}$ & 44.9256 & 74.8489 & 74.8489 \\
$\mathbf{R}_{7}$ & 51.0537 & 51.4227 & 66.9131 \\
$\mathbf{R}_{8}$ & 32.3244 & 33.0551 & 50.4844 \\
$\mathbf{R}_{9}$ & 19.8283 & 21.6756 & 31.9301 \\
$\mathbf{R}_{10}{ }^{a}$ & 22.0342 & 23.3132 & 31.4151 \\
\hline
\end{tabular}

${ }^{a}$ As a reference polymer, the optimized structure of $\mathbf{R}_{10}$ at the B3LYP/cc-pvDZ theory level is equilibrium because its three lowest frequencies are positive. 
Table S3. The orbital occupancy (in $e$ ) and orbital energy (in au) of $\mathbf{R}_{6}$ at the B3LYP/cc-pvDZ theory level. $\mathbf{R}=\left(\mathrm{C}_{3} \mathrm{~N}_{3}\right)_{2}$.

\begin{tabular}{|c|c|c|c|c|c|c|c|}
\hline No & Bond & Occupancy & Energy & No & Bond & Occupancy & Energy \\
\hline 1 & $\mathrm{~N} 1-\mathrm{C} 2$ & 1.984 & -0.87392 & 64 & C34-N35 & 1.631 & -0.34979 \\
\hline 2 & $\mathrm{~N} 1-\mathrm{C} 2$ & 1.661 & -0.34679 & 65 & C34-C68 & 1.967 & -0.65708 \\
\hline 3 & N1-C6 & 1.984 & -0.87426 & 66 & N35-C36 & 1.981 & -0.86237 \\
\hline 4 & $\mathrm{C} 2-\mathrm{N} 3$ & 1.981 & -0.86237 & 67 & N37-C38 & 1.981 & -0.88912 \\
\hline 5 & C2-C36 & 1.968 & -0.68238 & 68 & N37-C42 & 1.982 & -0.85993 \\
\hline 6 & N3-C4 & 1.983 & -0.88867 & 69 & N37-C42 & 1.662 & -0.35753 \\
\hline 7 & $\mathrm{~N} 3-\mathrm{C} 4$ & 1.631 & -0.34979 & 70 & C38-N39 & 1.983 & -0.88867 \\
\hline 8 & $\mathrm{C} 4-\mathrm{N} 5$ & 1.981 & -0.88912 & 71 & C38-N39 & 1.631 & -0.34979 \\
\hline 9 & C4-C38 & 1.967 & -0.65708 & 72 & N39-C40 & 1.981 & -0.86237 \\
\hline 10 & N5-C6 & 1.982 & -0.85993 & 73 & C40-N41 & 1.984 & -0.87392 \\
\hline 11 & N5-C6 & 1.662 & -0.35753 & 74 & C40-N41 & 1.661 & -0.34679 \\
\hline 12 & $\mathrm{C} 6-\mathrm{C} 8$ & 1.969 & -0.68055 & 75 & $\mathrm{C} 40-\mathrm{C} 72$ & 1.969 & -0.68141 \\
\hline 13 & N7-C8 & 1.984 & -0.87426 & 76 & N41-C42 & 1.984 & -0.87426 \\
\hline 14 & N7-C12 & 1.984 & -0.87392 & 77 & $\mathrm{C} 42-\mathrm{C} 46$ & 1.969 & -0.68141 \\
\hline 15 & N7-C12 & 1.661 & -0.34679 & 78 & N43-C44 & 1.981 & -0.88912 \\
\hline 16 & C8-N9 & 1.982 & -0.85993 & 79 & N43-C48 & 1.982 & -0.85993 \\
\hline 17 & C8-N9 & 1.662 & -0.35753 & 80 & N43-C48 & 1.662 & -0.35753 \\
\hline 18 & N9-C10 & 1.981 & -0.88912 & 81 & C44-N45 & 1.983 & -0.88867 \\
\hline 19 & C10-N11 & 1.983 & -0.88867 & 82 & C44-N45 & 1.631 & -0.34979 \\
\hline 20 & C10-N11 & 1.631 & -0.34979 & 83 & $\mathrm{~N} 45-\mathrm{C} 46$ & 1.981 & -0.86237 \\
\hline 21 & $\mathrm{C} 10-\mathrm{C} 44$ & 1.967 & -0.65708 & 84 & C46-N47 & 1.984 & -0.87392 \\
\hline 22 & N11-C12 & 1.981 & -0.86237 & 85 & C46-N47 & 1.661 & -0.34679 \\
\hline 23 & $\mathrm{C} 12-\mathrm{C} 14$ & 1.968 & -0.68238 & 86 & N47-C48 & 1.984 & -0.87426 \\
\hline 24 & N13-C14 & 1.984 & -0.87392 & 87 & C48-C52 & 1.969 & -0.68141 \\
\hline 25 & N13-C14 & 1.661 & -0.34679 & 88 & N49-C50 & 1.981 & -0.88912 \\
\hline 26 & N13-C18 & 1.984 & -0.87426 & 89 & N49-C54 & 1.982 & -0.85993 \\
\hline 27 & C14-N15 & 1.981 & -0.86237 & 90 & N49-C54 & 1.662 & -0.35753 \\
\hline 28 & N15-C16 & 1.983 & -0.88867 & 91 & C50-N51 & 1.983 & -0.88867 \\
\hline 29 & $\mathrm{~N} 15-\mathrm{C} 16$ & 1.631 & -0.34979 & 92 & C50-N51 & 1.631 & -0.34979 \\
\hline 30 & C16-N17 & 1.981 & -0.88912 & 93 & N51-C52 & 1.981 & -0.86237 \\
\hline 31 & $\mathrm{C} 16-\mathrm{C} 50$ & 1.967 & -0.65708 & 94 & C52-N53 & 1.984 & -0.87392 \\
\hline 32 & N17-C18 & 1.982 & -0.85993 & 95 & C52-N53 & 1.661 & -0.34679 \\
\hline 33 & N17-C18 & 1.662 & -0.35753 & 96 & N53-C54 & 1.984 & -0.87426 \\
\hline 34 & $\mathrm{C} 18-\mathrm{C} 20$ & 1.969 & -0.68141 & 97 & C54-C58 & 1.969 & -0.68141 \\
\hline 35 & N19-C20 & 1.981 & -0.86237 & 98 & N55-C56 & 1.981 & -0.88912 \\
\hline 36 & N19-C24 & 1.983 & -0.88867 & 99 & N55-C60 & 1.982 & -0.85993 \\
\hline 37 & $\mathrm{~N} 19-\mathrm{C} 24$ & 1.631 & -0.34979 & 100 & N55-C60 & 1.662 & -0.35753 \\
\hline 38 & $\mathrm{C} 20-\mathrm{N} 21$ & 1.984 & -0.87392 & 101 & C56-N57 & 1.983 & -0.88867 \\
\hline 39 & $\mathrm{C} 20-\mathrm{N} 21$ & 1.661 & -0.34679 & 102 & C56-N57 & 1.631 & -0.34979 \\
\hline 40 & $\mathrm{~N} 21-\mathrm{C} 22$ & 1.984 & -0.87426 & 103 & N57-C58 & 1.981 & -0.86237 \\
\hline 41 & C22-N23 & 1.982 & -0.85993 & 104 & C58-N59 & 1.984 & -0.87392 \\
\hline
\end{tabular}




\begin{tabular}{llll|llll}
42 & $\mathrm{C} 22-\mathrm{N} 23$ & 1.662 & -0.35753 & 105 & $\mathrm{C} 58-\mathrm{N} 59$ & 1.661 & -0.34679 \\
43 & $\mathrm{C} 22-\mathrm{C} 30$ & 1.969 & -0.68141 & 106 & $\mathrm{~N} 59-\mathrm{C} 60$ & 1.984 & -0.87426 \\
44 & $\mathrm{~N} 23-\mathrm{C} 24$ & 1.981 & -0.88912 & 107 & $\mathrm{C} 60-\mathrm{C} 64$ & 1.969 & -0.68141 \\
45 & $\mathrm{C} 24-\mathrm{C} 56$ & 1.967 & -0.65708 & 108 & $\mathrm{~N} 61-\mathrm{C} 62$ & 1.981 & -0.88912 \\
46 & $\mathrm{~N} 25-\mathrm{C} 26$ & 1.983 & -0.88867 & 109 & $\mathrm{~N} 61-\mathrm{C} 66$ & 1.982 & -0.85993 \\
47 & $\mathrm{~N} 25-\mathrm{C} 26$ & 1.631 & -0.34979 & 110 & $\mathrm{~N} 61-\mathrm{C} 66$ & 1.662 & -0.35753 \\
48 & $\mathrm{~N} 25-\mathrm{C} 30$ & 1.981 & -0.86237 & 111 & $\mathrm{C} 62-\mathrm{N} 63$ & 1.983 & -0.88867 \\
49 & $\mathrm{C} 26-\mathrm{N} 27$ & 1.981 & -0.88912 & 112 & $\mathrm{C} 62-\mathrm{N} 63$ & 1.631 & -0.34979 \\
50 & $\mathrm{C} 26-\mathrm{C} 62$ & 1.967 & -0.65708 & 113 & $\mathrm{~N} 63-\mathrm{C} 64$ & 1.981 & -0.86237 \\
51 & $\mathrm{~N} 27-\mathrm{C} 28$ & 1.982 & -0.85993 & 114 & $\mathrm{C} 64-\mathrm{N} 65$ & 1.984 & -0.87392 \\
52 & $\mathrm{~N} 27-\mathrm{C} 28$ & 1.662 & -0.35753 & 115 & $\mathrm{C} 64-\mathrm{N} 65$ & 1.661 & -0.34679 \\
53 & $\mathrm{C} 28-\mathrm{N} 29$ & 1.984 & -0.87426 & 116 & $\mathrm{~N} 65-\mathrm{C} 66$ & 1.984 & -0.87426 \\
54 & $\mathrm{C} 28-\mathrm{C} 32$ & 1.969 & -0.68055 & 117 & $\mathrm{C} 66-\mathrm{C} 70$ & 1.969 & -0.68141 \\
55 & $\mathrm{~N} 29-\mathrm{C} 30$ & 1.984 & -0.87392 & 118 & $\mathrm{~N} 67-\mathrm{C} 68$ & 1.981 & -0.88912 \\
56 & $\mathrm{~N} 29-\mathrm{C} 30$ & 1.661 & -0.34679 & 119 & $\mathrm{~N} 67-\mathrm{C} 72$ & 1.982 & -0.85993 \\
57 & $\mathrm{~N} 31-\mathrm{C} 32$ & 1.984 & -0.87426 & 120 & $\mathrm{~N} 67-\mathrm{C} 72$ & 1.662 & -0.35753 \\
58 & $\mathrm{~N} 31-\mathrm{C} 36$ & 1.984 & -0.87392 & 121 & $\mathrm{C} 68-\mathrm{N} 69$ & 1.983 & -0.88867 \\
59 & $\mathrm{~N} 31-\mathrm{C} 36$ & 1.661 & -0.34679 & 122 & $\mathrm{C} 68-\mathrm{N} 69$ & 1.631 & -0.34979 \\
60 & $\mathrm{C} 32-\mathrm{N} 33$ & 1.982 & -0.85993 & 123 & $\mathrm{~N} 69-\mathrm{C} 70$ & 1.981 & -0.86237 \\
61 & $\mathrm{C} 32-\mathrm{N} 33$ & 1.662 & -0.35753 & 124 & $\mathrm{C} 70-\mathrm{N} 71$ & 1.984 & -0.87392 \\
62 & $\mathrm{~N} 33-\mathrm{C} 34$ & 1.981 & -0.88912 & 125 & $\mathrm{C} 70-\mathrm{N} 71$ & 1.661 & -0.34679 \\
63 & $\mathrm{C} 34-\mathrm{N} 35$ & 1.983 & -0.88867 & 126 & $\mathrm{~N} 71-\mathrm{C} 72$ & 1.984 & -0.87426 \\
\hline & & & & & & &
\end{tabular}


TABLE S4: The Bond Path Lengths (BPL, in $\AA$ ), the Ellipticities $(\varepsilon)$, the Charge Densities ( $\rho$ in $e /$ Bhor $^{2}$ ), the Laplacian of $\rho\left(\nabla^{2} \rho\right)$ of the Bonds or Intramolecular Interactions in $\mathrm{R}_{n}$ at the B3LYP/cc-pvDZ Theory Level. $\mathrm{R}=\left(\mathrm{C}_{3} \mathrm{~N}_{3}\right)_{2}, n=7-9$.

\begin{tabular}{|c|c|c|c|c|}
\hline Bond path & Length $(\AA)$ & $\varepsilon$ & $\rho$ & $\nabla^{2} \rho$ \\
\hline \multicolumn{5}{|l|}{$\mathbf{R}_{7}$} \\
\hline N3...N79 & 3.170 & 20.931 & 0.008 & 0.027 \\
\hline N13...N37 & 3.174 & 22.613 & 0.008 & 0.027 \\
\hline N19...N43 & 3.173 & 32.753 & 0.008 & 0.027 \\
\hline N55...N63 & 3.172 & 24.543 & 0.008 & 0.027 \\
\hline N69...N73 & 3.175 & 26.33 & 0.008 & 0.027 \\
\hline \multicolumn{5}{|l|}{$\mathbf{R}_{8}$} \\
\hline N3...N85 & 2.997 & 0.64 & 0.011 & 0.035 \\
\hline N9...N31 & 2.997 & 0.65 & 0.011 & 0.035 \\
\hline N13...N37 & 2.998 & 0.647 & 0.011 & 0.035 \\
\hline N19...N43 & 2.995 & 0.637 & 0.011 & 0.035 \\
\hline N27...N49 & 2.994 & 0.627 & 0.011 & 0.035 \\
\hline N55...N75 & 2.994 & 0.633 & 0.011 & 0.035 \\
\hline N63...N91 & 2.995 & 0.638 & 0.011 & 0.035 \\
\hline N69...N79 & 2.996 & 0.639 & 0.011 & 0.035 \\
\hline \multicolumn{5}{|l|}{$\mathbf{R}_{\mathbf{9}}$} \\
\hline N5...N9 & 2.316 & 2.234 & 0.034 & 0.202 \\
\hline N13...N37 & 2.911 & 0.106 & 0.012 & 0.039 \\
\hline N19...N43 & 2.912 & 0.113 & 0.012 & 0.039 \\
\hline N29...N99 & 2.959 & 0.596 & 0.012 & 0.038 \\
\hline N33...N71 & 2.954 & 0.575 & 0.012 & 0.039 \\
\hline N49...N57 & 2.315 & 2.351 & 0.035 & 0.203 \\
\hline N63...N103 & 2.881 & 0.036 & 0.014 & 0.042 \\
\hline N81...N97 & 3.146 & 4.003 & 0.008 & 0.027 \\
\hline N79...C80 & 7.556 & 120684.71 & 0 & 0 \\
\hline
\end{tabular}


Table S5. The time consumption (in Hour) of polymers in the processes of structure and frequency optimization at the specified theory levels

\begin{tabular}{|c|c|c|c|c|c|c|c|}
\hline & $\mathbf{R}_{3}$ & $\mathbf{R}_{4}$ & $\mathbf{R}_{5}$ & $\mathbf{R}_{6}$ & $\mathbf{R}_{7}$ & $\mathbf{R}_{8}$ & $\mathbf{R}_{\mathbf{9}}$ \\
\hline Opt B3LYP/cc-pvDZ & $0.4 / 3$ & $0.5 / 3$ & $1 / 6$ & $2 / 13$ & $56 / 223$ & $6.2 / 1033$ & $128 / 573$ \\
\hline Freq $\quad$ B3LYP /cc-pvDZ & $13 / 13$ & $29 / 30$ & $37 / 37$ & $48 / 48$ & $195 / 195$ & $100 / 137$ & $325 / 376$ \\
\hline Opt B3LYP /cc-pvTZ ${ }^{\mathrm{a} / \mathrm{b}}$ & $13 /-$ & $8 /-$ & $24 /-$ & $34 /-$ & $768 /-$ & & \\
\hline Freq $\quad$ B3LYP $/ c c-p v T Z$ & $218 /-$ & $448 /-$ & $543 /-$ & $741 /-$ & $2211 /-$ & & \\
\hline Opt B3LYP /aug-cc-pvDZ ${ }^{\mathrm{a} / \mathrm{b}}$ & $7 / 97$ & $10 / 727$ & $2745^{\mathrm{c}} /-$ & $351^{\mathrm{c} /-}$ & & & \\
\hline Freq B3LYP/aug-cc-pvDZ & $169 / 283$ & $267 / 767$ & - & - & & & \\
\hline Opt wB97XD/aug-cc-pvDZ ${ }^{a / b}$ & $14 / 196$ & $12 /-$ & $1456^{c /-}$ & & & & \\
\hline Freq $\quad$ wB97XD /aug-cc-pvDZ & $218 / 332$ & $291 /-$ & - & & & & \\
\hline Opt $\quad$ MP2/aug-cc-pvDZ ${ }^{a / b}$ & $364 /-$ & $2041 /-$ & & & & & \\
\hline Freq $\quad$ MP2 /aug-cc-pvDZ & $5098 \mathrm{~d} /-$ & $1730^{\mathrm{d}} /-$ & & & & & \\
\hline Opt $\quad$ B3LYP $/ 6-31+G(d)^{a / b}$ & $6583 \%$ & & & & & & \\
\hline
\end{tabular}

a Time consumption by using the optimized structure at the B3LYP/cc-pvDZ level as the initial (input) structures.

${ }^{\mathrm{b}}$ Time consumption by using the structures (no symmetry) optimized at MD (molecular mechanics) as the initial (input) structures.

${ }^{\mathrm{c}}$ Convergence failure and run terminated at this time point.

${ }^{\mathrm{d}}$ Frequency optimization terminates at this time point after exhausting $512 \mathrm{~GB}$ of memory and $1280 \mathrm{~GB}$ of disk space for RWF file.

- Convergence failure and run terminated, or unable to operation. 